\title{
ACO-Initialized Wavelet Neural Network for Vibration Fault Diagnosis of Hydroturbine Generating Unit
}

\author{
Zhihuai Xiao, ${ }^{1}$ Xinying He, ${ }^{1}$ Xiangqian Fu, ${ }^{1}$ and O. P. Malik ${ }^{2}$ \\ ${ }^{1}$ School of Power and Mechanical Engineering, Wuhan University, Wuhan, Hubei 430072, China \\ ${ }^{2}$ Department of Electrical and Computer Engineering, University of Calgary, Calgary, AB, Canada T2N 1N4 \\ Correspondence should be addressed to Zhihuai Xiao; xiaozhihuai@126.com
}

Received 24 November 2014; Accepted 12 January 2015

Academic Editor: Yun-Bo Zhao

Copyright (c) 2015 Zhihuai Xiao et al. This is an open access article distributed under the Creative Commons Attribution License, which permits unrestricted use, distribution, and reproduction in any medium, provided the original work is properly cited.

\begin{abstract}
Considering the drawbacks of traditional wavelet neural network, such as low convergence speed and high sensitivity to initial parameters, an ant colony optimization- (ACO-) initialized wavelet neural network is proposed in this paper for vibration fault diagnosis of a hydroturbine generating unit. In this method, parameters of the wavelet neural network are initialized by the ACO algorithm, and then the wavelet neural network is trained by the gradient descent algorithm. Amplitudes of the frequency components of the hydroturbine generating unit vibration signals are used as feature vectors for wavelet neural network training to realize mapping relationship from vibration features to fault types. A real vibration fault diagnosis case result of a hydroturbine generating unit shows that the proposed method has faster convergence speed and stronger generalization ability than the traditional wavelet neural network and ACO wavelet neural network. Thus it can provide an effective solution for online vibration fault diagnosis of a hydroturbine generating unit.
\end{abstract}

\section{Introduction}

Nowadays, hydroturbine generating units are becoming larger, more complicated, and more integrated, which not only makes regulation and operation of the hydroturbine generating unit complicated but also increases the probability of occurrence of faults. Therefore, it is of great significance to research effective fault diagnosis methods that give early alert before faults happen or avoid deterioration of existing faults resulting in great economic losses.

About $80 \%$ of the hydroturbine generating unit faults reveal characteristics in vibration signals [1]. Vibration signals of a hydroturbine generating unit, a complicated and nonlinear system, are generally influenced by multiple hydraulic, mechanical, and electrical/electronic factors [2]. These factors may interact with each other, which makes it difficult to construct by theoretical analysis a one-to-one relationship between the vibration feature and the cause of the fault. All these factors lead to the difficulty of fault diagnosis for a hydroturbine generating unit.
Taking the characteristics of hydroturbine generating unit vibration signals into consideration, nonlinear diagnosis models are often utilized to realize effective mapping from vibration feature sets to fault sets [3-6]. Neural network [7] has perfect self-organization, adaptive learning, and remembrance abilities. It can realize complicated relationship mapping in nonlinear systems and has become a dominant method in the area of hydroturbine generating unit fault diagnosis. Feedforward neural network trained by back propagation method is one of the most widely used methods [3-5]. However, such a neural network has drawbacks, for example, slow convergence speed and inclination to be trapped in local minima [8]. Wavelet neural network, a kind of feedforward network proposed in 1992 [9] on the basis of wavelet analysis theory by substituting excitation function for wavelet function, has developed fast [10-12]. However, even though by combining time-frequency localization ability of wavelet analysis and self-learning ability of neural network a wavelet neural network has strong approximation, fault tolerance, and classification abilities, it cannot avoid drawbacks of 
slow convergence speed and high sensitivity to initialization parameters [13]. Therefore, optimizing parameters of wavelet neural networks by heuristic optimization algorithms is becoming an important research topic [14-16].

Ant colony optimization (ACO) algorithm is one kind of the heuristic optimization algorithms. It has perfect global optimization characteristics, strong robust ability, and great distributed computing system. ACO wavelet neural network, which uses ACO to learn parameters of wavelet neural network, preserves the advantages of ACO and does not have the drawbacks of sensitivity to initializing parameters. However, its training speed is still slow according to the information in the literature. Therefore, an ACO-initialized wavelet neural network is proposed in this paper and used in the vibration fault diagnosis of a hydroturbine generating unit. This method employs ACO to train the parameters of a wavelet network and the obtained parameters are taken as the initialization parameters. Vibration frequency features of a hydroturbine generating unit are taken as the inputs and fault types are taken as the outputs of the wavelet neural network. A fault diagnosis model of the hydroturbine generating unit based on ACO-initialized wavelet neural network is constructed. Fault diagnosis results show that, compared with the traditional wavelet neural network and ACO wavelet neural network, not only can the method proposed in this paper increase the speed of convergence but it also has strong generalization ability.

\section{Vibration Types and Characteristics of Hydroturbine Generating Units}

According to disturbing force types of vibration signals, vibration types can be divided into hydraulic vibration, mechanical vibration, and electrical vibration [2].

2.1. Hydraulic Vibration. Hydraulic vibration is caused by water flow and machinery. There are many factors which lead to this kind of vibration such as hydraulic imbalance, draft tube pressure pulsation, nonuniformity in the path of circulating water flow, nonuniform gap of runner wearing ring, Karman vortex street, clearance jet, cavity erosion, and wrong in cam relationship. The characteristic of this type of vibration is that vibration frequency is different for each vibration source.

2.2. Mechanical Vibration. Mechanical vibration is aroused by improper installation of the unit, drawbacks of the unit structure, or damage in the component of the running unit. There are many factors which lead to this kind of vibration such as imbalance of the rotating part of the unit, misalignment of the axis, defects in the bearing, rotor-tostator rub, and looseness of the connection. The characteristic of this kind of vibration is that vibration frequency is the rotation frequency or a multiple of the rotation frequency.

2.3. Electrical Vibration. Electrical vibration is caused by nonuniformity of magnetic flux density, unbalance of electromagnetic pull, and stator core looseness. There are many factors which lead to this kind of vibration such as the rotor pole coil turn-to-turn short circuit, nonuniform air gap between rotor and stator, wrong polarity order of the core, out of round of rotor inside or stator outside, and unbalance of current among three phases. The characteristic of this kind of vibration is that vibration frequency is the rotation frequency or the frequency of the polar in the hydropower generator.

It can be seen from the above that vibration signals are the synthesis of results aroused by hydraulic vibration, mechanical vibration, and electrical vibration. It has highly nonlinear characteristic. Hydroturbine generating unit fault diagnosis based on neural network method is used to extract features of these vibration signals and neural network is used to map these features to corresponding fault type in order to realize the fault diagnosis for hydroturbine generating unit.

\section{Wavelet Neural Network}

Wavelet neural network is a kind of neural network that is constructed based on wavelet analysis theory. As wavelet analysis theory ensures the $L^{2}(R)$ approximation ability, wavelet function can substitute for excitation function of the neural network to form a new kind of feedforward neural network model.

3.1. Structure of Wavelet Neural Network. Structure of a wavelet neural network is shown in Figure 1, where $M, n$, and $N$ are number of input layer nodes, hidden layer nodes, and output layer nodes, respectively. $\mathbf{x}\left(x_{k}, k=1,2, \ldots, M\right)$, $\mathbf{o}\left(o_{j}, j=1,2, \ldots, n\right)$, and $\mathbf{y}\left(y_{i}, i=1,2, \ldots, N\right)$ are vectors of input layer, hidden layer, and output layer, respectively. $W_{j k}(j=1,2, \ldots, n ; k=1,2, \ldots, M)$ is weight parameter between input layer and hidden layer. $V_{i j}(i=1,2, \ldots, N$; $j=1,2, \ldots, n)$ is weight parameter between hidden layer and output layer. $\mathbf{b}\left(b_{j}, j=1,2, \ldots, n\right)$ and $\mathbf{a}\left(a_{j}, j=\right.$ $1,2, \ldots, n)$ are translation parameters and scaling parameters, respectively. $h$ is wavelet function.

Suppose that net ${ }_{j}=\sum_{k=1}^{M} W_{j k} x_{k}$; then the model of wavelet network can be expressed as

$$
y_{i}=\sum_{j=1}^{n} V_{i j} h\left(\frac{\text { net }_{j}-b_{j}}{a_{j}}\right) .
$$

3.2. Training Method of Wavelet Neural Network. Suppose that the error function of a wavelet neural network is

$$
E=\frac{1}{2} \sum_{l=1}^{L} \sum_{i=1}^{N}\left(d_{i}^{l}-y_{i}^{l}\right)^{2}
$$

Here, $L$ is the number of training samples and $\mathbf{d}\left(d_{i}, i=\right.$ $1,2, \ldots, N)$ is the object output vector. 


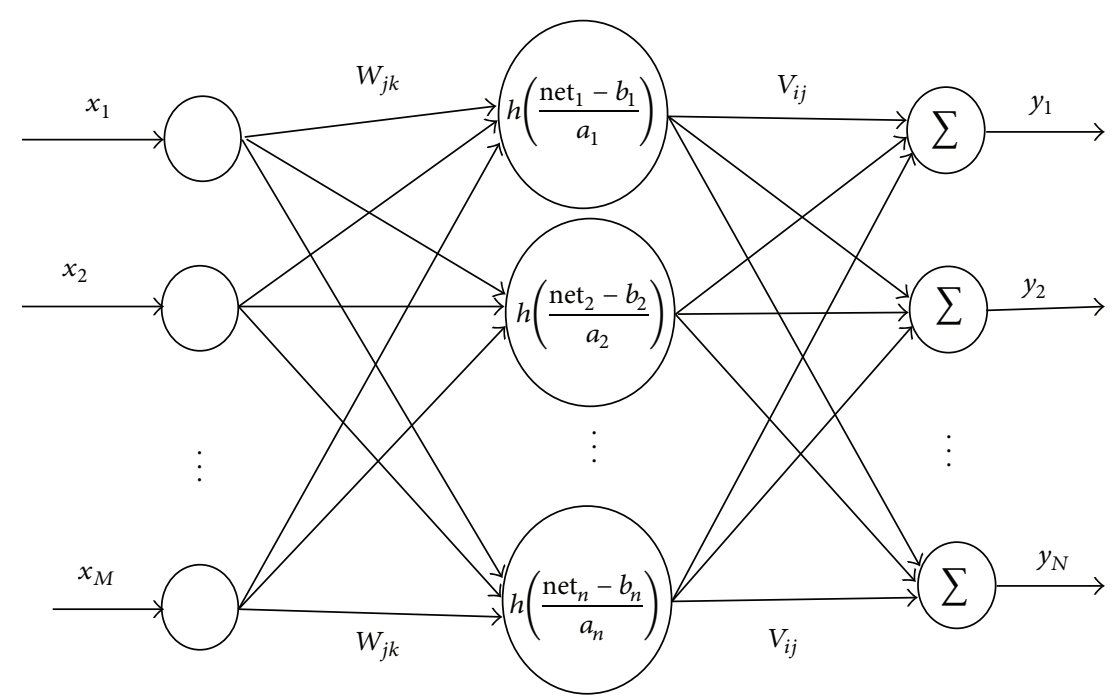

FIGURE 1: Structure of wavelet neural network.

Generally, the training method of a wavelet neural network is the gradient descent method, for which the equations for parameters adjustment are shown in

$$
\begin{aligned}
V_{i j}^{\text {new }} & =V_{i j}^{\text {old }}-\eta \frac{\partial E}{\partial V_{i j}} \\
W_{j k}^{\text {new }} & =W_{j k}^{\text {old }}-\eta \frac{\partial E}{\partial W_{j k}} \\
a_{j}^{\text {new }} & =a_{j}^{\text {old }}-\eta \frac{\partial E}{\partial a_{j}} \\
b_{j}^{\text {new }} & =b_{j}^{\text {old }}-\eta \frac{\partial E}{\partial b_{j}} .
\end{aligned}
$$

Here, $\eta$ is learning factor. Gradient of parameters are shown in

$$
\begin{gathered}
\frac{\partial E}{\partial V_{i j}}=-\sum_{l=1}^{L}\left(d_{i}^{l}-y_{i}^{l}\right) h\left(\frac{\text { net }_{j}^{l}-b_{j}}{a_{j}}\right) \\
\frac{\partial E}{\partial W_{j k}}=-\sum_{l=1}^{L} \sum_{i=1}^{N}\left(d_{i}^{l}-y_{i}^{l}\right) V_{i j} h^{\prime}\left(\frac{\text { net }_{j}^{l}-b_{j}}{a_{j}}\right) \frac{1}{a_{j}} x_{k}^{l} \\
\frac{\partial E}{\partial a_{j}}=\sum_{L=1}^{l} \sum_{i=1}^{N}\left(d_{i}^{l}-y_{i}^{l}\right) V_{i j} h^{\prime}\left(\frac{\text { net }_{j}^{l}-b_{j}}{a_{j}}\right) \frac{\left(\text { net }_{j}^{l}-b_{j}\right)}{a_{j}^{2}} \\
\frac{\partial E}{\partial b_{j}}=\sum_{l=1}^{L} \sum_{i=1}^{N}\left(d_{i}^{l}-y_{i}^{l}\right) V_{i j} h^{\prime}\left(\frac{\text { net }_{j}^{l}-b_{j}}{a_{j}}\right) \frac{1}{a_{j}} .
\end{gathered}
$$

\section{ACO-Initialized Wavelet Neural Network}

4.1. Theory of ACO-Initialized Wavelet Neural Network. ACO algorithm [15] is a kind of heuristic global optimization algorithm which takes ant seeking food theory as its basic theory and seeks the optimization in the solution space of an objective function. Pheromone is the intermediary by which ants communicate with each other. When an ant seeks food, it will secrete pheromone to mark trace and determine forward direction according to the amount of pheromone on the trace which has direct ratio relationship with the number of ants which have passed this trace. At the beginning, ants will choose trace randomly. Because the number of ants in a shorter trace is larger than in a longer one, more pheromone will be left in the shorter trace and hence the probability of the shorter trace being chosen will be larger. This is the positive feedback mechanism. As the iteration times increases, the whole ant colony will eventually restrain itself to a shorter trace which represents the optimized solution.

The specific theory of ACO-initialized wavelet neural network is that the parameters waiting to be initialized are treated as the nodes in the seeking traces, and then all of the ants need to choose the nodes to reach the food source. During the food seeking process, error function of the wavelet neural network is taken as the evaluation function to adjust the amount of pheromone and guide the direction of the ants.

4.2. Procedure of ACO-Initialization of the Wavelet Neural Network. The procedure of ACO-initialization of the wavelet neural network is similar to the procedure of ACO wavelet neural network [15]. The difference is that the former method employs ACO algorithm to train the neural network and then the parameters after the initial training are taken as the initialization parameters of the wavelet neural network. The initial training time is not very large and does not need to reach certain error precision. The error precision of the wavelet network is determined by the further training process. In the latter method, ACO algorithm is used to train the wavelet neural network until the error precision or certain training time is reached.

Suppose there are $m$ parameters waiting to be initialized, which include weight parameters, scaling parameters, and 


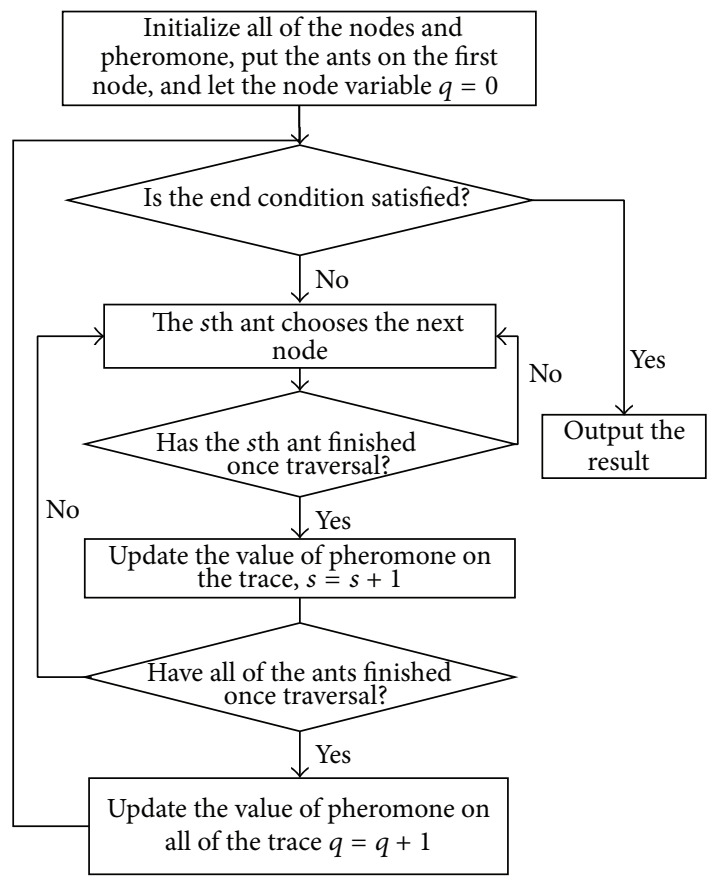

FIGURE 2: Flowchart of ACO-initialized wavelet network.

translation parameters. Then, these parameters are lined up and form a vector, $\mathbf{P}\left(P_{i}, i=1,2, \ldots, m\right)$, in which $K$ random numbers corresponding to each of the elements are chosen to form $m$ sets $I_{P i}(i=1,2, \ldots, m)$. Suppose the total number of ants is $S$. Each ant takes off from $I_{P i}$ first. Then it chooses elements from each of the sets independently and randomly according to the law of probability transfer. The ant will reach the food source when all of the sets are chosen. After that, the pheromone of each set will be adjusted. This process is repeated until the error precision or certain training time is reached.

Flowchart of the ACO-initialized wavelet neural network is shown as in Figure 2. The specific procedure is as follows.

(1) Relevant parameters initialization: set pheromone of each element in set $I_{P i}$ as $\tau_{j}\left(I_{P i}\right)(0)=C(j=1,2, \ldots, K)$.

(2) Determine whether the stop condition (the error precision or certain training time) is reached or not. If so, output the optimization solution and stop the iteration; otherwise turn to the third step.

(3) The $j$ th element is chosen from set $I_{P i}$ according to the law of probability transfer shown as follows for ant $s(s=$ $1,2, \ldots, S)$ :

$$
\operatorname{Prob}\left(\tau_{j}^{s}\left(I_{P i}\right)\right)=\frac{\tau_{j}\left(I_{P i}\right)}{\sum_{u=1}^{K} \tau_{u}\left(I_{P i}\right)} .
$$

(4) After the sth ant finished all of the nodes in the chosen process, the chosen parameters will be input into the wavelet neural network and the error calculated. Then calculate the amount of pheromone corresponding to the sth ant and the $j$ th nodes:

$$
\Delta \tau_{j}^{s}\left(I_{P i}\right)= \begin{cases}\frac{Q}{E^{s}} \quad \begin{array}{l}
\text { The } s \text { th ant chooses the } j \text { th elements } \\
\text { in set } I_{P i} \text { during this circulation } \\
0 \quad \text { otherwise }
\end{array}\end{cases}
$$

where $Q$ is a constant which is used to adjust the pheromone adjustment speed and $E^{s}$ is the error calculated by inputting the parameters into wavelet neural network after the sth ant finished all of the nodes traversal.

(5) After all of the ants finished choosing once, adjust all of the elements in set $I_{P i}$ according to the following equation, record the optimized value, and turn to step (2):

$$
\tau_{j}\left(I_{P i}\right)(t+m)=\rho \tau_{j}\left(I_{P i}\right)(t)+\Delta \tau_{j}\left(I_{P i}\right) .
$$

Here, $\rho(0<\rho<1)$ represents the durability of pheromone and $1-\rho$ represents the disappearing degree between $t$ and $t+m$.

\section{Hydroturbine Generating Fault Diagnosis Example}

5.1. Selection of Fault Features. Vibration signals of a hydroturbine generating unit are the synthesized reflection of hydraulic, mechanical, and electric vibration factors and so on. The method, which takes amplitude of vibration signals frequency components as feature vector and employs neural network to realize the mapping from vibration feature set to fault set, is a common method for hydroturbine generating unit fault diagnosis. In this paper, the amplitudes of vibration signals frequency components $0.4 \sim 0.5 f, 1 f, 2 f, 3 f$, and $>3 f$ are chosen to form the feature vector, and 3 fault conditions (vortex with eccentric, unbalance and misalignment) and normal condition of hydroturbine generating unit are taken as fault types waiting to be recognized. Here, the letter " $f$ " represents the fundamental frequency of hydroturbine generating unit. ACO-initialized wavelet neural network is employed to diagnose vibration fault of the hydroturbine generating unit. Three-layer model, namely, input layer, hidden layer, and output layer, is selected to form the structure of the wavelet neural network. To make the structure simpler and the result of the neural network more intuitive, multi-input and single-output structure is employed. According to the characteristics of the training samples, the number of input nodes is chosen as 5 which is equal to the number of feature parameters; the number of output nodes is 1 . The number of hidden layer nodes is chosen as 8 according to experience. Define the objective values 1, 2, 3 , and 4 as the values corresponding to vortex with eccentric, unbalance, misalignment, and normal machinery conditions, respectively. Choose 2 groups of feature samples for each of the 4 conditions as training samples and 1 group of feature samples for each of the 4 conditions as testing samples. The normalized training and testing feature samples are shown in Tables 1 and 2 , respectively $[5,8]$. 
TABLE 1: Training samples.

\begin{tabular}{|c|c|c|c|c|c|c|}
\hline Fault types & $(0.4-0.5) f$ & $1 f$ & $2 f$ & $3 f$ & $>3 f$ & Object values \\
\hline Vortex with eccentric & 0.88 & 0.22 & 0.02 & 0.04 & 0.06 & 1 \\
\hline Vortex with eccentric & 0.85 & 0.25 & 0.06 & 0.02 & 0.01 & 1 \\
\hline Unbalance & 0.04 & 0.98 & 0.10 & 0.02 & 0.02 & 2 \\
\hline Unbalance & 0.03 & 0.96 & 0.12 & 0.04 & 0.03 & 2 \\
\hline Misalignment & 0.02 & 0.41 & 0.43 & 0.34 & 0.15 & 3 \\
\hline Misalignment & 0.02 & 0.45 & 0.42 & 0.28 & 0.29 & 3 \\
\hline Normal & 0.01 & 0.02 & 0.01 & 0.05 & 0.04 & 4 \\
\hline Normal & 0.10 & 0.03 & 0.02 & 0.03 & 0.04 & 4 \\
\hline
\end{tabular}

TABLE 2: Testing samples.

\begin{tabular}{lcccccc}
\hline Fault types & $(0.4-0.5) f$ & $1 f$ & $2 f$ & $3 f$ & Object values \\
\hline Vortex with eccentric & $\mathbf{0 . 8 2}$ & $\mathbf{0 . 2 8}$ & $\mathbf{0 . 0 5}$ & $\mathbf{0 . 0 4}$ & $\mathbf{0 . 0 3}$ & 0.02 \\
Unbalance & 0.02 & 0.91 & 0.08 & 0.01 & 0.20 & $\mathbf{1}$ \\
Misalignment & 0.01 & 0.48 & 0.48 & 0.36 & 0.04 & 3 \\
Normal & 0.10 & 0.03 & 0.02 & 0.03 & 0.04 \\
\hline
\end{tabular}

TABLE 3: Diagnosis results of three methods.

\begin{tabular}{|c|c|c|c|c|}
\hline Fault types & Wavelet neural network & ACO wavelet neural network & $\begin{array}{c}\text { ACO-initialized wavelet neural } \\
\text { network }\end{array}$ & Object values \\
\hline Vortex with eccentric & 1.1073 & 1.0925 & 1.0955 & 1 \\
\hline Unbalance & 1.8307 & 2.1806 & 2.0564 & 2 \\
\hline Misalignment & 2.7499 & 2.8566 & 2.9255 & 3 \\
\hline Normal & 3.9745 & 3.9700 & 3.9723 & 4 \\
\hline
\end{tabular}

5.2. Model Parameters Selection and Diagnosis Results. In this example, the number of iterations is set as 10 , the number of ants in an ant colony is set as 10, Q is set as $1, \rho$ is set as 0.6 , and the random number $N$ is set as 20 . Wavelet basis function is set as commonly used Morlet wavelet function $h(t)=\cos (1.75 t) \exp \left(-(1 / 2) t^{2}\right)$, learning factor $\eta$ is set as 0.05 , and the objective error $E$ is set as 0.01 .

In order to prove the effectiveness of the ACO-initialized wavelet neural network, ACO wavelet neural network and traditional wavelet neural network are chosen as comparison methods in the same computer and with the same parameters. Figures 3 and 4 show the training line of the traditional wavelet neural network and the ACO-initialized wavelet neural network, respectively. It can be seen from these figures that the traditional neural network needs 958 training times to reach the objective error, while the ACO-initialized wavelet neural network needs only 63 training times to reach the objective error.

Using the testing samples in Table 2 to test the obtained ACO-initialized wavelet neural network, the ACO wavelet neural network, and the traditional wavelet neural network, Table 3 shows the test results.

Table 4 demonstrates the diagnosis error and training time of the three kinds of networks. Here, the training time of the ACO-initialized wavelet neural network is the sum of 10 times ACO initializing training time (0.4531s) and
TABLE 4: Error and training time of three methods.

\begin{tabular}{lccc}
\hline $\begin{array}{l}\text { Diagnosing } \\
\text { methods }\end{array}$ & $\begin{array}{c}\text { Wavelet neural } \\
\text { network }\end{array}$ & $\begin{array}{c}\text { ACO wavelet } \\
\text { neural network }\end{array}$ & $\begin{array}{c}\text { ACO-initialized } \\
\text { wavelet neural } \\
\text { network }\end{array}$ \\
\hline $\begin{array}{l}\text { Diagnosing } \\
\text { error }\end{array}$ & $\mathbf{0 . 0 5 1 7}$ & $\mathbf{0 . 0 3 1 3}$ & $\mathbf{0 . 0 0 9 3}$ \\
$\begin{array}{l}\text { Training } \\
\text { time/s }\end{array}$ & 2.3438 & 9.3281 & 0.6094 \\
\hline
\end{tabular}

wavelet neural network training time $(0.1563 \mathrm{~s})$. The unit of the numbers in Table 4 is second.

5.3. Analysis of the Diagnosis Results. According to Tables 3 and 4 and Figures 3 and 4, analysis of the results can be obtained as follows.

(1) All three kinds of neural networks can recognize fault types of the hydroturbine generating unit.

(2) Compared with the traditional neural network, ACO wavelet neural network has higher generalization ability, but it needs more computer time to finish the training process.

(3) The training time of the ACO-initialized wavelet neural network is $0.6094 \mathrm{~s}$, and its diagnosing error 


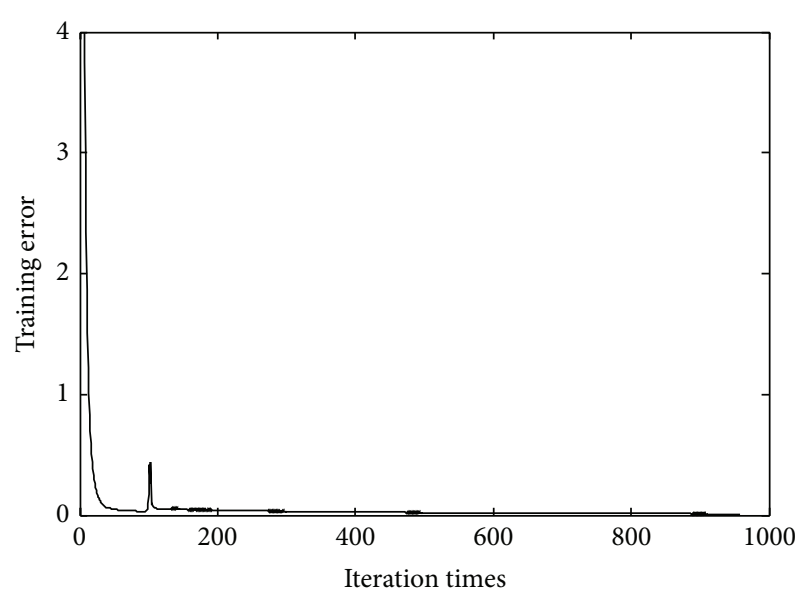

FIGURE 3: Training curve of wavelet network.

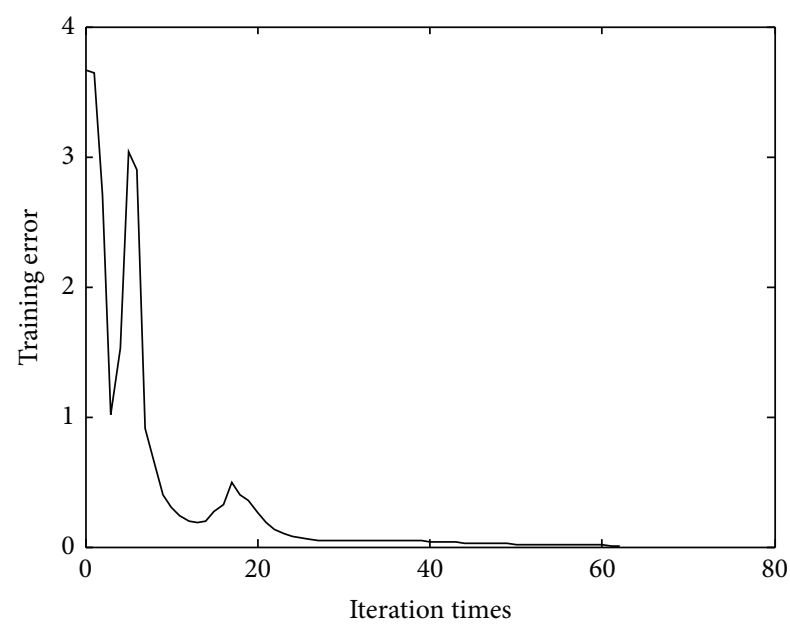

FIgURE 4: Training curve of ACO-initialized wavelet network.

is 0.0093 . Compared with the other two types of neural networks, the proposed network not only has higher generalization ability but also increases the convergence speed. Thus, the ACO-initialized wavelet neural network is more suited to online vibration fault diagnosis for hydroturbine generating unit.

\section{Conclusions}

Wavelet neural network is sensitive to initial parameters. In other words, when the parameters are initialized improperly, the convergence speed of the neural network will become slower and its generalization ability will become worse. This constrains its application in the hydroturbine generating unit fault diagnosis. In this paper, the advantages of both ACO algorithm and wavelet neural network are combined and the parameters optimized by the ACO algorithm are used as the initialized parameters of the wavelet neural network. The wavelet neural network is trained further and the trained wavelet neural network is applied to vibration fault diagnosis of a hydroturbine generating unit. The method proposed in this paper can determine the initial parameters of the wavelet neural network and also has the time-frequency location property of wavelet neural network and global optimization ability of the ACO algorithm. By using the extracted features of amplitude of frequency components of hydroturbine generating unit vibration signals, the traditional wavelet neural network, the ACO wavelet neural network, and the ACOinitialized wavelet neural network are compared with each other. The results show that the ACO-initialized wavelet neural network has stronger generalization ability and faster convergence speed and thus is more suitable to diagnose the vibration faults of a hydroturbine generating unit online.

\section{Conflict of Interests}

The authors declare that there is no conflict of interests regarding the publication of this paper.

\section{Acknowledgment}

The authors acknowledge the supports from the National Natural Science Foundation of China under Grant no. 51379160 .

\section{References}

[1] D.-L. Zhao, W. Ma, W.-K. Liang, and X.-Q. Luo, "Data fusion fault diagnosis and simulation of hydroelectric units vibration," Proceedings of the Chinese Society of Electrical Engineering, vol. 25, no. 20, pp. 137-142, 2005 (Chinese).

[2] D. Shen, F. Chu, and S. Chen, "Diagnosis and identification of vibration accident for hydro-generator unit," Journal of Hydrodynamics, vol. 15, no. 1, pp. 129-133, 2000 (Chinese).

[3] X. Fu, G.-L. Liu, and J. Jiang, "Application of BP neural networks to condition monitoring and fault diagnosis system of hydrogenerator units," Engineering Journal of Wuhan University, vol. 35, no. 1, pp. 24-28, 2002 (Chinese).

[4] R. Jia, L. Bai, X. Luo, and F. Liu, "Expert system on fault diagnosis based on neural network for hydropower units," Journal of Hydroelectric Engineering, vol. 23, no. 6, pp. 120-123, 2004.

[5] X. Yang, J. Xie, and C. Sun, "Neural network method for vibration fault diagnosis of hydroelectric generating set," Journal of Hydraulic Engineering, supplement 1, pp. 94-97, 1998 (Chinese).

[6] W. Peng, P. Guo, and X. Luo, "Research on vibration fault diagnosis of hydro-turbine generating unit based on LS-SVM and information fusion technology," Journal of Hydroelectric Engineering, vol. 26, no. 6, pp. 137-142, 2007.

[7] D. Zhang and L. Yu, "Exponential state estimation for Markovian jumping neural networks with time-varying discrete and distributed delays," Neural Networks, vol. 35, pp. 103-111, 2012.

[8] W. Peng and X. Luo, "Research on vibration fault diagnosis of hydro-turbine generating unit based on wavelet neural network," Journal of Hydroelectric Engineering, vol. 26, no. 1, pp. 123-128, 2007 (Chinese).

[9] Q. Zhang and A. Benveniste, "Wavelet networks," IEEE Transactions on Neural Networks, vol. 3, no. 6, pp. 889-898, 1992.

[10] R. Cheng and Y. Bai, "A novel approach to fuzzy wavelet neural network modeling and optimization," International Journal of Electrical Power \& Energy Systems, vol. 64, pp. 671-678, 2015. 
[11] Z. Zainuddin and O. Pauline, "Modified wavelet neural network in function approximation and its application in prediction of time-series pollution data," Applied Soft Computing Journal, vol. 11, no. 8, pp. 4866-4874, 2011.

[12] J. Cao, Z. Lin, and G.-B. Huang, "Composite function wavelet neural networks with extreme learning machine," Neurocomputing, vol. 73, no. 7-9, pp. 1405-1416, 2010.

[13] T.-S. Wang and L.-P. Zhang, "Application of immune wavelet network model to fault diagnosis of hydro-turbine generating units," Journal of Hydraulic Engineering, vol. 40, no. 6, pp. 762767, 2009 (Chinese).

[14] L. Zuo, L.-G. Hou, D.-M. Gao, X.-H. Peng, and W.-C. Wu, "Fault diagnosis of analog circuit based on PSO-WNN," Journal of Beijing University of Technology, vol. 36, no. 3, pp. 306-309, 2010 (Chinese).

[15] D. Feng, Application Research of Ant Colony Algorithm and Wavelet Network in Complexity Science, Tianjin University, Tianjin, China, 2008, (Chinese).

[16] L. Liu, Y. Li, and W. Wang, "Fault diagnosis study on the vibration of hydropower units based on GA neural network and evidence theory fusion," Journal of Hydroelectric Engineering, vol. 27, no. 5, pp. 163-167, 2008. 


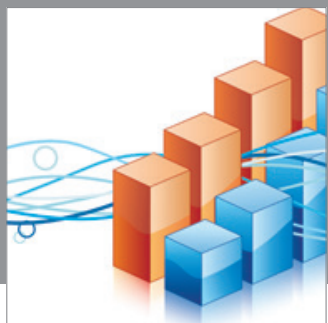

Advances in

Operations Research

mansans

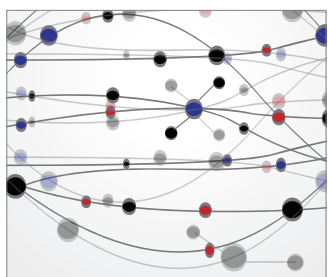

The Scientific World Journal
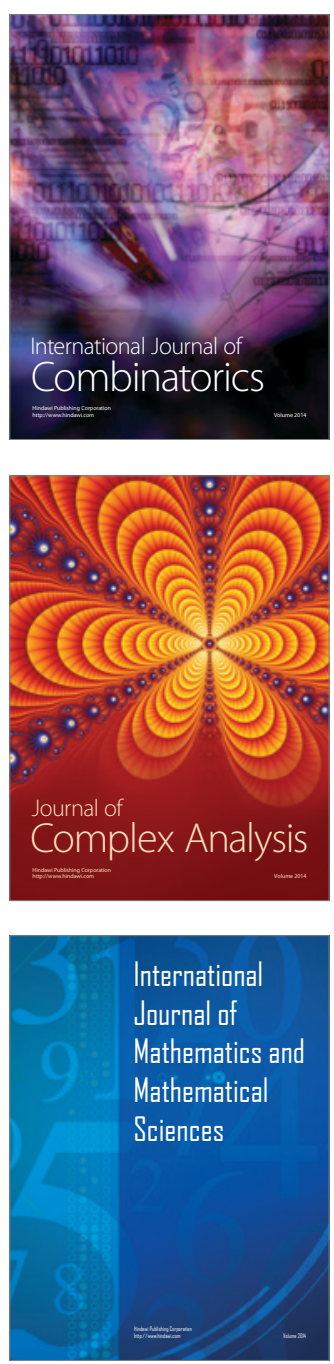
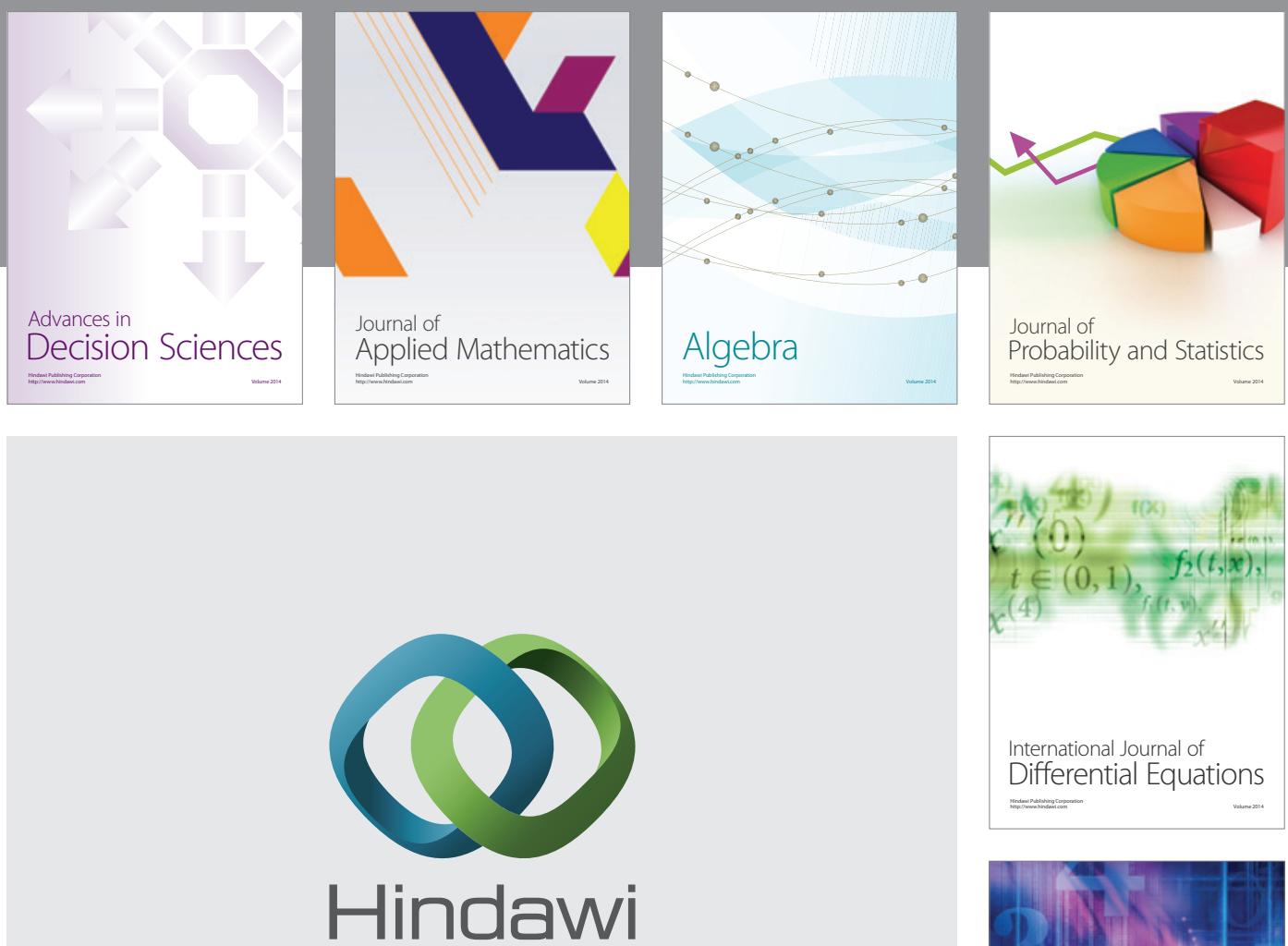

Submit your manuscripts at http://www.hindawi.com
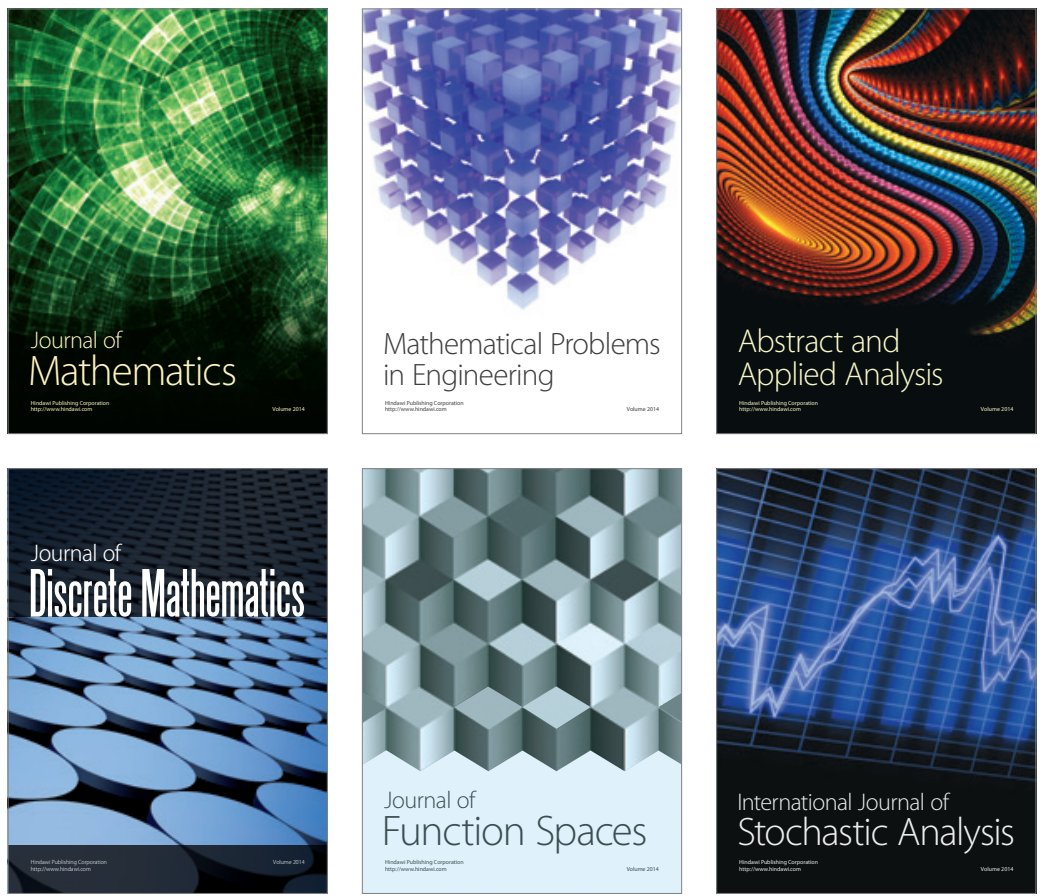

Journal of

Function Spaces

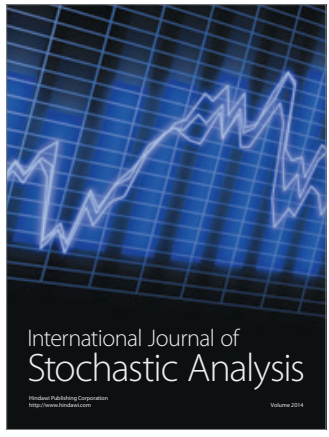

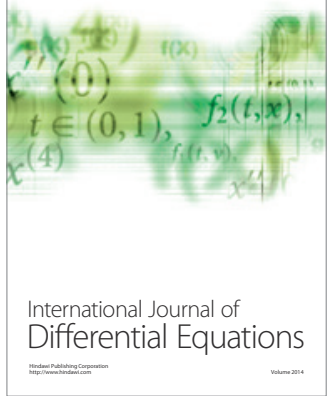
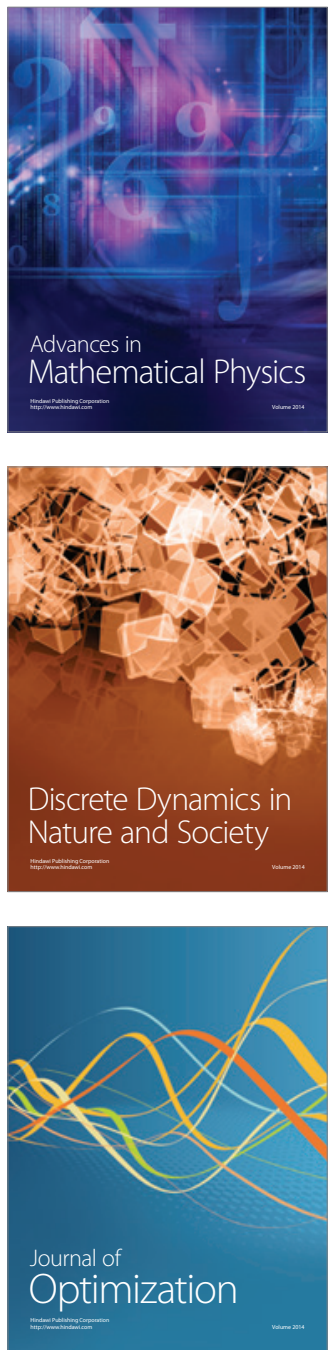